\title{
Film Thickness Formation in Nanoscale due to Effects of Elastohydrodynamic, Electrostatic and Surface force of Solvation and Van der Waals
}

\author{
M.F. Abd Al-Samieh a \\ a Mechanical design \& production Department, Military Technical College, Cairo, Egypt.
}

Keywords:

Ultra-thin films

Elastohydrodynamics

Solvation

Van der Waals' force

Electrostatic force

\section{Corresponding author:}

M.F. Abd Al-Samieh

Mechanical design \& production

Department, Military Technical

College, Cairo, Egypt.

E-mail: mohamed.fahmy203@hotmail.com

\begin{abstract}
A B S T R A C T
The mechanism of oil film with a thickness in the nanoscale is discussed in this paper. A polar lubricant of propylene carbonate is used as the intervening liquid between contiguous bodies in concentrated contacts. A pressure caused by the hydrodynamic viscous action in addition to double layer electrostatic force, Van der Waals inter-molecular forces, and solvation pressure due to inter-surface forces is considered in calculating the ultrathin lubricating films. The numerical solution has been carried out, using the Newton-Raphson iteration technique, applied for the convergence of the hydrodynamic pressure. The results show that, at separations beyond about five molecular diameters of the intervening liquid, the formation of a lubricant film thickness is governed by combined effects of viscous action and surface force of an attractive Van der Waals force and a repulsive double layer force. At smaller separations below about five molecular diameters of the intervening liquid, the effect of solvation force is dominant in determining the oil film thickness
\end{abstract}

(C) 2017 Published by Faculty of Engineering

\section{INTRODUCTION}

Recent advances in micro-engineering have resulted in the downsizing of machine elements with very thin film conjunctions, typically in the order of or below $200 \mathrm{~nm}$ and micro/nanotribology has also significantly advanced [1-4]. When the applied load is very small and the lubricant film is very thin, the hydrodynamic viscous force becomes comparable with the surface force and thus it must be taken into consideration in solving lubrication problems [5-7]. An example of this is made by the computer magnetic slider/disk engineers who are considering reducing the flying height of the slider over the disk to ten nanometers or less in order to increase the recording density of the disk.

When the solid surfaces reach the distance of several nanometers, continuum theories of attractive Van der Waals and repulsive doublelayer forces often describe their interaction. Israelachvili [8-9] and Israelachvili and McGuiggan [10] have measured the force between two crossed cylinders immersed in a 
fluid and found that if the lubricant is polar, the force at large separations is well described by the DLVO theory (i.e. the action of Van der Waals and repulsive double-layer forces together). Christensen and Horn [11] have presented measurements of force between two curved mica surfaces in propylene carbonate. They found that above $8 \mathrm{~nm}$ and out to $50 \mathrm{~nm}$, the force law is accurately described by double-layer theory and below $8 \mathrm{~nm}$ the Van der Waals attraction reduces the net force below the double layer as expected from the DLVO theory and below $3 \mathrm{~nm}$ the force is oscillatory.

Chan and Horn [12] have presented measurements of oil film thickness as a function of time as the liquid films are squeezed between two molecularly smooth mica surfaces. They found that Reynolds' theory of hydrodynamic lubrication seems to be applicable down to a film thickness of $50 \mathrm{~nm}$. They also found that, for thinner films the drainage of the fluid film is slower than that theoretically predicted.

Matsuoka and Kato [13] and Al-Samieh and Rahnejat [14] have developed a new method for calculating the total pressure; as a combined effect of EHL and the molecular surface force of Van der Waals and solvation forces. From their results, they found that the surface elastic deformation due to surface force cause a thicker lubricant film, and cannot be neglected any more when compared to the ordinary fluid viscous force. An interesting conclusion from their result is that, the lubrication film thickness agrees well with the conventional lubrication theory in case of the films larger than $5 \mathrm{~nm}$, and deviation from the theoretical prediction and discretization of the film thickness is observed in case of a film thickness of several nanometers. They also found that their calculation results agree well with their experimental data [15-17].

Although, the aforementioned investigation was successful to show the effect of surface force of solvation and Van der Waals on the formation of ultra-thin lubricating films in the case where the intervening liquid is non-polar, but in case of polar lubricant an additional force of repulsive double-layer should be aided. This paper attempts to contribute to the on-going process of understanding of ultra-thin film lubrication formation by investigating the effect of surface force action of solvation, Van der Waals as well as electrostatic force, where the intervening liquid between the two planner surfaces is polar.

\section{BACKGROUND THEORY}

In the conventional EHL theory, film thickness and pressure distribution are obtained by simultaneous solution of the Reynolds' equation, the elastic film shape, incorporating the contact deformation of the semi-infinite solid (given by the elasticity equation) and the load balance equation. However, in the case of ultra-thin film thickness a pressure caused by the Van der Waals inter-molecular forces, solvation pressure due to inter-surface forces and double layer electrostatic force should be considered. The total pressure; $P$, is composed of four components, solvation pressure; $P_{s}$, Van der Waals pressure contribution; $P_{v d w}$, double layer electrostatic force; $P_{e}$, and conventional viscous pressure; $P_{h}$ :

$$
P=P_{s}+P_{v d w}+P_{e}+P_{h}
$$

The total pressure in equation (1) is calculated simultaneously with the elastic film shape equation in the same manner as that carried out for the conventional solution to the elastohydrodynamic lubrication problem.

\subsection{Elastohydrodynamic Pressure}

The dimensionless Reynolds' equation for point contact condition under steady-state entraining can be written as:

$$
\frac{\partial}{\partial X}\left(\frac{\bar{\rho} H^{3}}{\bar{\eta}} \frac{\partial P_{h}}{\partial X}\right)+\frac{1}{K^{2}} \frac{\partial}{\partial Y}\left(\frac{\bar{\rho} H^{3}}{\bar{\eta}} \frac{\partial P_{h}}{\partial Y}\right)=\lambda \frac{\partial}{\partial X}(\bar{\rho} H)
$$

Where the following dimensionless variables apply:

$X=x / b, Y=y / a, \bar{\eta}=\eta / \eta_{0}, \bar{\rho}=\rho / \rho_{0}, \quad H=h R_{x} / b^{2}$, $P_{h}=p_{h} / p_{\text {Her }}$

and: $\lambda=\frac{12 u \eta_{o} R_{x}^{2}}{b^{3} P_{H e r}}$

The variation in density of the lubricant with pressure is defined by Dowson and Higginson [18] as:

$$
\bar{\rho}=1+\frac{\varepsilon P_{h} P_{H e r}}{1+\xi P_{h} P_{H e r}}
$$


Where $\varepsilon$ and $\zeta$ are constants, dependent upon the type of lubricant used. The variation in the viscosity of the lubricant with pressure in dimensionless form is given by Roelands [19] as:

$$
\left.\bar{\eta}=\exp \left[\ln \eta_{\circ}+9.67\right]\left(1+5.1 * 10^{-9} P_{h} P_{H e r}\right)^{z}-1\right]
$$

where:

$$
\mathrm{Z}=\frac{\alpha}{5.1 * 10^{-9}\left[\ln \eta_{\circ}+9.67\right]}
$$

The elastic film shape in dimensionless form is assumed to be of the same as that reported by Hamrock and Dowson [20], given by:

$$
H(X, Y)=H_{\circ}+\frac{(X-m)^{2}}{2}+\frac{K^{2} R_{x}}{R_{y}} \frac{(Y-l)^{2}}{2}+\frac{R_{x} \delta(X, Y)}{b^{2}}
$$

where, the dimensional elastic deformation at any point $\mathrm{x}, \mathrm{y}$ is defined by Hamrock and Dowson [20] as:

$$
\delta_{I, J}(x, y)=\frac{2}{\Pi} \sum_{j=1}^{n y} \sum_{i=1}^{n x} P_{i, j} D_{i^{*}, j^{*}}
$$

where:

$$
i^{*}=|I-i|+1, j^{*}=|J-j|+1
$$

The Newton-Raphson method is applied for the solution of the Reynolds'equation in the following numerical form:

$$
\sum_{l=2}^{m y-1 m x-1} \sum_{k=2}^{i, j} J_{k, l} \Delta \bar{P}_{k, l}=-F_{i, j}
$$

where, the Jacobian matrix is a tensorial quantity, given in terms of the residual derivatives as:

$$
J_{k, l}^{i, j}=\frac{\partial F_{i . j}}{\partial P_{k, l}}
$$

Using the Gauss-Seidel iteration method, the system state equation can be written as:

$$
\begin{aligned}
& \Delta P_{k, l}^{n}=\left(-F_{i, j}-J_{k-1, l}^{i, j} \Delta P_{k-1, l}^{n}-J_{k+1, l}^{i, j} \Delta P_{k+1, l}^{n-1}\right. \\
& \left.-J_{k, l-1}^{i, j} \Delta P_{k, l-1}^{n}-J_{k, l+1}^{i, j} \Delta P_{k, l+1}^{n-1}\right) / J_{k, l}^{i, j}
\end{aligned}
$$

where $\mathrm{n}$ is the iteration counter in the above recursive equation.

For the reason of good numerical stability an under-relaxation factor is employed to update the pressure according to:

$$
P_{i, j}^{n}=P_{i, j}^{n-1}+\Omega \Delta P_{i, j}^{n}
$$

where $\Omega$ is the under-relaxation factor, typically chosen as 0.01 under the reported conditions in this paper.
The convergence criterion on the pressure is:

$$
\left[\frac{\sum_{i} \sum_{j}\left(\bar{P}_{i, j}^{n}-\bar{P}_{i, j}^{n-1}\right)^{2}}{N}\right]^{0.5} \leq 10^{-4}
$$

The convergence criterion on load balance is given as:

$$
\left|\iint P(X, Y) d X d Y-\frac{2}{3} \pi\right| \leq 10^{-4}
$$

\subsection{Molecular and Surface actions in narrow conjunctions}

The net interaction between two surfaces involves both the electrostatic double layer force and the Van der Waals force. The Van der Waals force dominates when the separation between the surfaces is small. It is also rather insensitive on the concentration of the electrolytes. On the other hand, the electrostatic double layer repulsion is strong at larger separations, and it is quite sensitive on the concentration of electrolytes. At small surface separations, less than a few molecular diameters the continuum theories of attractive Van der Waals and repulsive electrostatic forces often fail to describe their interaction and the effect of solvation force is dominant in determining the oil film thickness.

\section{a- Solvation Pressure}

Solvation force (i.e. structural force) is a surface interaction force that acts between two solid surfaces, when they approach each other to form a very small gap filled by a fluid. Israelachvili et al [7], Israelachvili [9], Horn and Israelachvili [21], Van Megen and Snook [22], and Homola et al [23] have studied the solvation force in the narrow contact of contiguous bodies. They have all shown that these surface forces have generally a decaying oscillatory characteristic as a function of gap (i.e. the film thickness). They vary as attractive and repulsive forces, with a periodicity equal to the mean diameter of the fluid molecules. Such oscillatory forces arise from the molecular geometry and local structure of the liquid medium, and reflect the forced ordering of the liquid molecules into discrete layers, when constrained between two surfaces.

The solvation pressure is obtained as: 


$$
P_{s}=-C e^{\frac{-h}{a}} \cos (2 \pi h / a)
$$

\section{b- Van der Waals Pressure}

Van der Waals' forces of attraction exist between two surfaces, when they are separated by a very thin fluid film (see for example, lifshitz [24]). Israelachvili [9] gives the pressure in the fluid, induced by the Van der Waals' forces as a function of separation as:

$$
P_{v d w}=\frac{-A}{6 \pi h^{3}}
$$

\section{c- Electrostatic forces between surfaces}

The electrostatic interactions are based on the socalled Poisson-Boltzmann equation, as described by Verwey and Overbeek [25]. They showed that the electrostatic repulsion between two charged surfaces or particles decays roughly exponentially with the distance separation between them. Israelachvili [9] showed that, the repulsive electrostatic pressure between two planar surfaces can be written as:

$$
p_{\text {elest }}=64 K T \rho_{\infty} \chi^{2} e^{-\kappa h}
$$

where, $\chi=\tanh \left(z e \psi_{o} / 4 K T\right), z$ is the valency, $e$ is the electronic charge, $\psi_{o}$ is the electrostatic surface potential, $K$ is the Boltzmann constant, $T$ is the temperature in degrees Kelvin, $\rho_{\infty}$ is the electrolyte concentration in the bulk and $\kappa$ is the Debye length.

At low surface potentials, below about $25 \mathrm{mV}$, the above equation [13] can be simplified as shown by Israelachvili [9] to:

$$
p_{\text {elest }}=2 \varepsilon \varepsilon_{o} \kappa^{2} \omega_{0}^{2} e^{-\kappa h}
$$

Where, $\varepsilon$ is the dielectric constant of the liquid (for propylene carbonate $=65$ ), $\varepsilon_{o}$ is the dielectric permittivity of the free space (8.854X10-12 (farads/meter), $k$ is the Debye length and $\omega_{0}$ is the surface potential.

\section{RESULTS AND DISCUSSION}

The predictions from the current numerical analysis have been compared with the numerical investigations carried out by Kato and Matsuoka
[17]. Lubricant and material properties used are given in Table 1.

Table 1. Physical and geometrical properties of contacting materials and OMCTS lubricant.

\begin{tabular}{|c|c|c|c|}
\hline $\begin{array}{c}\text { Viscosity } \\
\boldsymbol{\eta}_{\mathbf{o}}\end{array}$ & $2.35 \mathrm{mPa} . \mathrm{s}$ & $\begin{array}{c}\text { Pressure of } \\
\text { viscosity } \\
\text { coefficient } \boldsymbol{\alpha}\end{array}$ & $10 \mathrm{GPa}^{-1}$ \\
\hline $\boldsymbol{\varepsilon}$ & $5.83 \times 10^{-10 \mathrm{~Pa}}$ & $\xi$ & $1.68 \times 10^{-9} \mathrm{~Pa}$ \\
\hline $\begin{array}{c}\text { Molecular } \\
\text { diameter, } \\
\boldsymbol{a}\end{array}$ & $1 \mathrm{~nm}$ & $\begin{array}{c}\text { Radius of } \\
\text { curvature of } \\
\text { solid A in } \mathbf{~ - ~} \\
\text { direction }\end{array}$ & $\begin{array}{c}R_{A x}=0.01111 \\
(\mathrm{~m})\end{array}$ \\
\hline $\begin{array}{c}\text { Young's } \\
\text { modulus } \\
\boldsymbol{E}_{\boldsymbol{A}}\end{array}$ & $34.5 \mathrm{GPa}$ & $\begin{array}{c}\text { Radius of } \\
\text { curvature of } \\
\text { solid A in y- } \\
\text { direction }\end{array}$ & $\begin{array}{c}R_{A y}=0.01111 \\
(\mathrm{~m})\end{array}$ \\
\hline $\begin{array}{c}\text { Young's } \\
\text { modulus } \\
\boldsymbol{E}_{\boldsymbol{B}}\end{array}$ & $34.5 \mathrm{GPa}$ & $\begin{array}{c}\text { Radius of } \\
\text { curvature of } \\
\text { solid B in } \mathbf{x}- \\
\text { direction }\end{array}$ & $R_{B x}=\infty(\mathrm{m})$ \\
\hline $\begin{array}{c}\text { Poisson's } \\
\text { ratio } \boldsymbol{v}_{\boldsymbol{A}}\end{array}$ & 0.205 & $\begin{array}{c}\text { Poisson's ratio } \\
\boldsymbol{v}_{\boldsymbol{B}}\end{array}$ & 0.205 \\
\hline
\end{tabular}

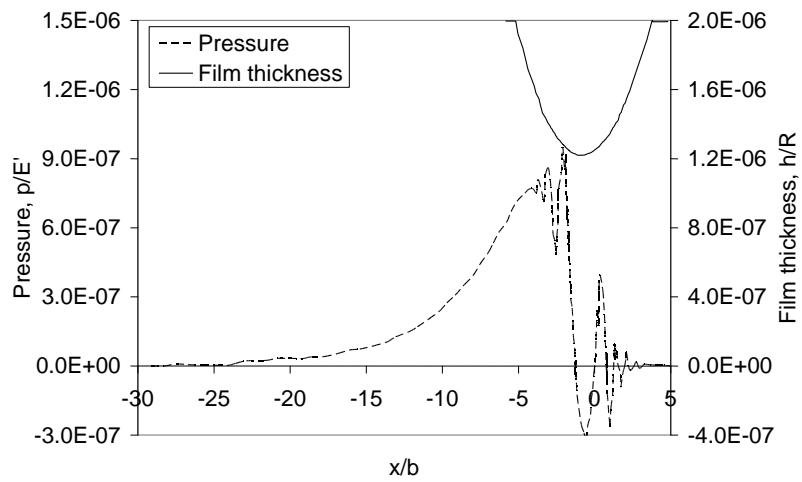

a) After Kato and Matsuoka [17]

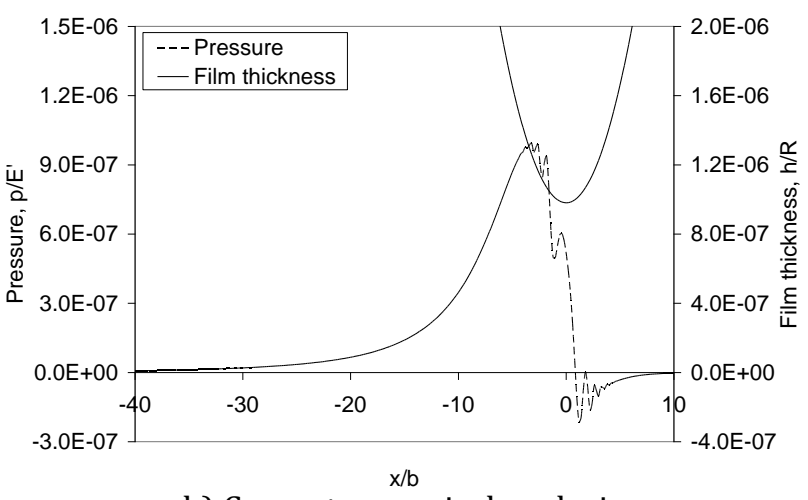

b) Current numerical analysis

Fig. 1. Total pressure profile and film shape for $W^{*}$ $=8.330 \times 10^{-12}$ and $U^{*}=6.525 \times 10^{-16}$.

Figure 1 shows the total pressure distribution and the corresponding lubricant film thickness in the direction of entraining motion through the central film, obtained for the model parameters; $\mathrm{a}=1 \mathrm{~nm}, \mathrm{C}=174 \mathrm{MPa}, \mathrm{A}=10^{-19}$ Joules and for the governing conditions: $\mathrm{W}^{*}=8.330 \times 10^{-12}$ (i.e. 30 
$\mu \mathrm{N}$ ), $\mathrm{U}^{*}=6.525 \times 10^{-16}$ (i.e. $100 \mu \mathrm{m} / \mathrm{s}$ ) and $\mathrm{G}^{*}=$ 360. Those depicted by Fig. 1(a) are the results obtained by Kato and Matsuoka [17] whilst those illustrated in Fig. 1(b) are obtained in the current analysis. According to Greenwood chart [26], the sliding condition of this example falls into Rigid-Isoviscos region where the elastic deformation is negligible. As can be observed, very good agreement exists between both sets of results. In fact the minimum film thickness is obtained as $9.8 \mathrm{~nm}$ in the current numerical analysis and as $9 \mathrm{~nm}$ in reference [17]. It is shown that the oscillatory solvation pressure lies on the conventional hydrodynamic viscous pressure. The surface deformation due to the solvation pressure was small namely the flattened centre and the occurrence of minimum film thickness at the trailing edge were not obtained under this sliding condition.

In the conditions described above the non-polar lubricant of Octamethylcyclotetrasiloxane (OMCTS) was used as the intervening media, thus there would have been no effect from the action of electrostatic force and only the Van der Waals force and molecular force of solvation is included. To observe the contributions of the electrostatic force in the formation of lubricant film thickness, the polar lubricant (propylene carbonate (PC)) was used as intervening media.

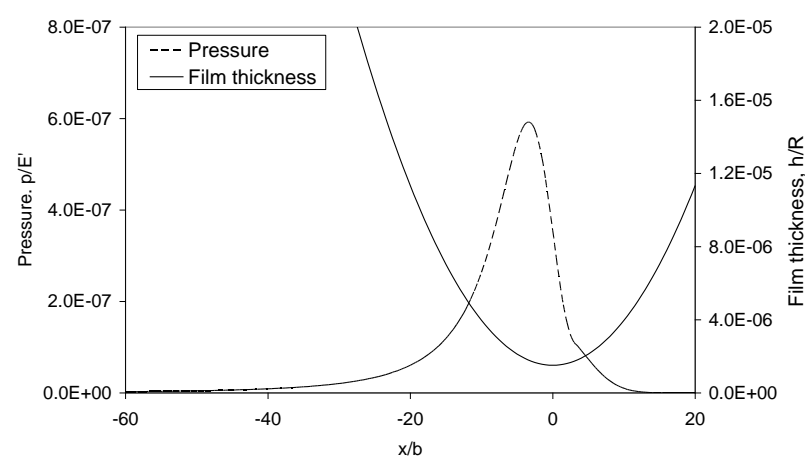

Fig. 2. Total pressure profile and film shape in the central line of contact as the result of combined viscous action and Van der Waals and the electrostatic double layer forces for $\mathrm{W}^{*}=8.330 \mathrm{X} 10^{-12}$ and $\mathrm{U}^{*}=7.775 \times 10^{-16}$.

Figure 2 shows the total pressure distribution and the corresponding elastic film shape for the central line of contact for the formation of lubricant film thickness due to the combined effect of hydrodynamic viscous action and the effect of Van der Waals and the electrostatic double layer forces (i.e. the action of DLVO theory). The model parameters used are $\mathrm{a}=0.5$ $\mathrm{nm}, \quad \mathrm{A}=5.51 \times 10^{-20}$ Joules. The governing conditions are: $\mathrm{W}^{*}=8.330 \times 10^{-12}$ (i.e. $30 \mu \mathrm{N}$ ), $\mathrm{U}^{*}=$ 7.775X10-16 (i.e. $100 \mu \mathrm{m} / \mathrm{s}$ ) and $\mathrm{G}^{*}=360$. Lubricant and material properties are provided in Table 2.

Table 2. Physical and geometrical properties of contacting materials and PC lubricant.

\begin{tabular}{|c|c|c|c|}
\hline Viscosity $\eta_{0}$ & $2.8 \mathrm{mPa} . \mathrm{s}$ & $\begin{array}{l}\text { Pressure of } \\
\text { viscosity } \\
\text { coefficient } \alpha\end{array}$ & $10 \mathrm{GPa}^{-1}$ \\
\hline$\varepsilon$ & $5.83 \times 10-10 \mathrm{~Pa}$ & $\xi$ & $1.68 \times 10^{-9} \mathrm{~Pa}$ \\
\hline $\begin{array}{l}\text { Molecular } \\
\text { diameter, } a\end{array}$ & $0.5 \mathrm{~nm}$ & $\begin{array}{c}\text { Radius of } \\
\text { curvature of } \\
\text { solid } A \text { in } x- \\
\text { direction }\end{array}$ & $\begin{array}{c}R_{A x}=0.01111 \\
(\mathrm{~m})\end{array}$ \\
\hline $\begin{array}{l}\text { Young's } \\
\text { modulus } E_{A}\end{array}$ & $34.5 \mathrm{GPa}$ & $\begin{array}{c}\text { Radius of } \\
\text { curvature of } \\
\text { solid } A \text { in } y- \\
\text { direction }\end{array}$ & $\begin{array}{c}R_{A y}=0.01111 \\
(\mathrm{~m})\end{array}$ \\
\hline $\begin{array}{c}\text { Young's } \\
\text { modulus } E_{\mathrm{B}}\end{array}$ & $34.5 \mathrm{GPa}$ & $\begin{array}{l}\text { Radius of } \\
\text { curvature of } \\
\text { solid B in } x- \\
\text { direction }\end{array}$ & $R_{B x}=\infty(\mathrm{m})$ \\
\hline $\begin{array}{l}\text { Poisson's } \\
\text { ratio } v_{A} \\
\end{array}$ & 0.205 & $\begin{array}{c}\text { Poisson's } \\
\text { ratio } v_{\mathrm{B}}\end{array}$ & 0.205 \\
\hline
\end{tabular}

In particular these conditions exhibit the contribution of the electrostatic pressure and relate to iso-viscous rigid regime in the Greenwood's chart [26]. The overall minimum lubricant film thickness obtained under these conditions is $16.31 \mathrm{~nm}$, whereas the numerical value for hydrodynamic action alone is $13.29 \mathrm{~nm}$ (Fig. 3) and that obtained using Brewe et al. [27] formula for circular point contacts under isoviscous rigid regime of lubrication is $12.6 \mathrm{~nm}$. However, as can be shown, the overall film thickness obtained due to the effect of electrostatic pressure is larger than those predicted by the formula, because they do not take into account the dominant regime of lubrication, which is due to the electrostatic forces. It can be seen that the effect of Van der Waals and the electrostatic double layer forces action causes the film thickness to be increased by about $23 \%$ of the actual film. Figure 4 shows the comparison between the formation of the oil film lubricant film thickness profile due to the action of hydrodynamic action alone, hydrodynamic viscous action and the effect of Van der Waals and the electrostatic double layer forces (i.e. the action of DLVO theory) and the effect of hydrodynamic viscous action and the action of DLVO theory and the solvation pressure. To see the effect of surface force action of solvation and Van der Waals forces on the formation of lubricant 
film thickness under these conditions, Fig. 5 shows the pressure distribution and the corresponding film thickness. In this case, the overall minimum oil film thickness is $16.31 \mathrm{~nm}$ which is been the same as that obtained numerically for hydrodynamic action and Van der Waals and the electrostatic double layer forces as shown in Fig. 2. Therefore, the effect of surface force of solvation is negligible and under these conditions, the effects of Van der Waals and the electrostatic double layer forces contributes to the generation of contact pressure.

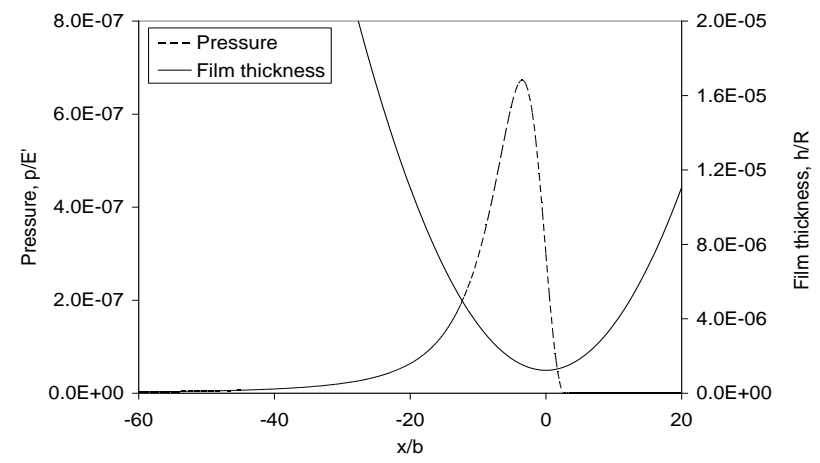

Fig. 3. Total pressure profile and film shape in the central line of contact as the result of viscous action alone for $\mathrm{W}^{*}=8.330 \times 10^{-12}$ and $\mathrm{U}^{*}=7.775 \times 10^{-16}$.

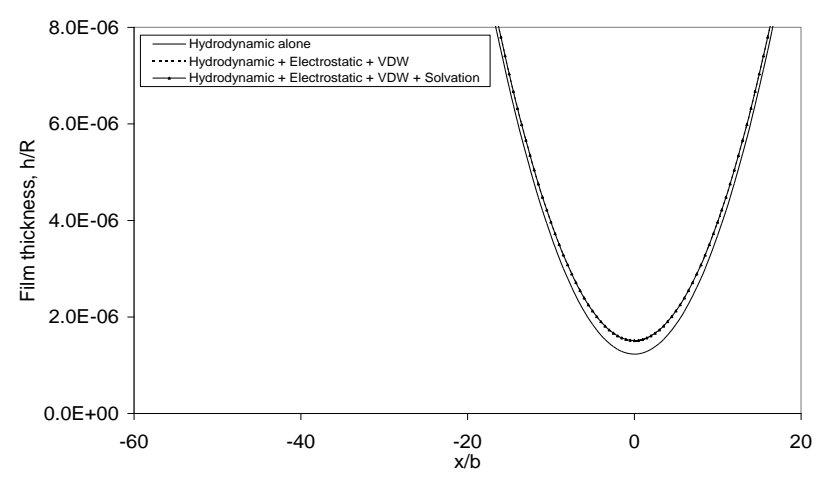

Fig. 4. Comparison between lubricants film thickness formation for $\mathrm{W}^{*}=8.330 \mathrm{X} 10^{-12}$ and $\mathrm{U}^{*}=7.775 \times 10^{-16}$.

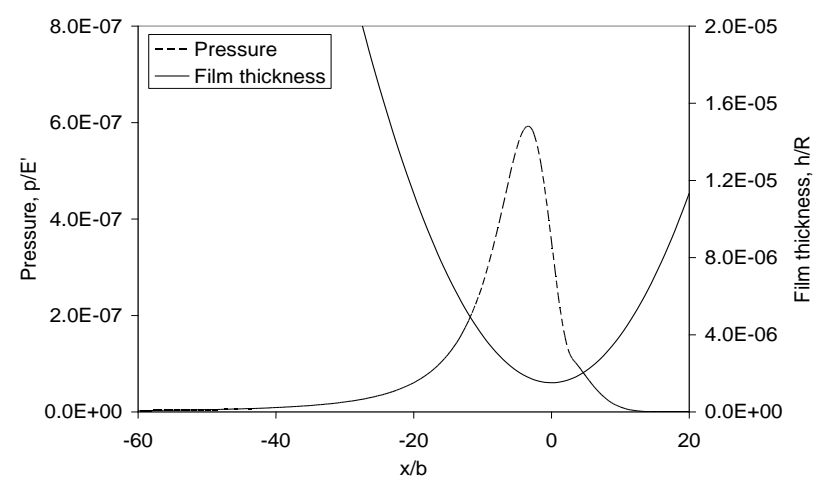

Fig. 5. Total pressure profile and film shape in the central line of contact as the result of combined viscous action and surface force of solvation and Van der Waals forces and electrostatic double layer forces for $\mathrm{W}^{*}=8.330 \times 10^{-12}$ and $\mathrm{U}^{*}=7.775 \mathrm{X} 10^{-16}$
For the same speed, but with an increased the applied load to $\mathrm{W}^{*}=8.330 \times 10^{-11}$, (i.e. $300 \mu \mathrm{N}$ ), the effect of solvation and Van der Waals pressures becomes significant, resulting in a lubricant film thickness of $1.31 \mathrm{~nm}$ as shown in Fig. 6. According to Greenwood chart [26], the sliding condition of this example falls into Elastic-Isoviscos region. The predicted minimum lubricant film thickness obtained due to hydrodynamic pressure alone is $0.534 \mathrm{~nm}$, and that obtained using Hamrock and Dowson [28] formula for circular point contacts under iso-viscous elastic regime of lubrication is $0.550 \mathrm{~nm}$ (the error is about $3 \%$ ). Under these conditions, it is clear that the dominant role of surface force of solvation and Van der Waals in determining the lubricant film thickness. It can be seen that an oscillatory distribution of the pressure due to solvation is observed. The effect of Van der Waals and the solvation forces causes the film thickness to be increased by about $60 \%$ compared to the formation of the film thickness for hydrodynamic action only. Under the previous operating conditions, the effect of the attractive Van der Waals force exceeds that the repulsive electrostatic double layer forces and the hydrodynamic action and the results is the two surfaces tend to stick to each other immediately. This case occurred if the Van der Waals and electrostatic double layer forces are added only forces to the hydrodynamic action without the effect of surface force of solvation. The physical explanation of this behaviour, is that the Van der Waals force vary as $\left(-1 / \mathrm{h}^{3}\right)$ while the electrostatic force vary as $\left(e^{-k h}\right)$, therefore, the Van der Waals forces always dominate at sufficiently small separations giving a force at molecular contact that is attractive (i.e. adhesion).

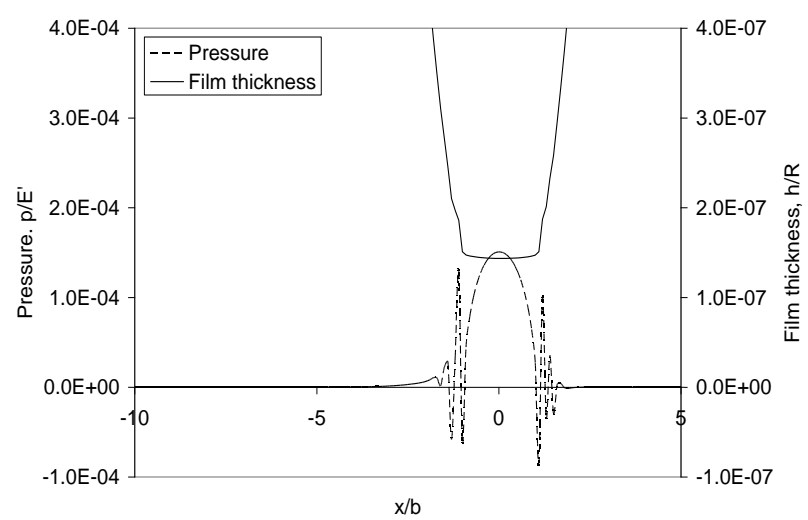

Fig. 6. Total pressure profile and film shape in the central line of contact as the result of combined viscous action and surface force of solvation and van der Waals forces for $\mathrm{W}^{*}=8.330 \times 10^{-11}$ and $\mathrm{U}^{*}=7.775 \times 10^{-16}$. 
Generally, the net interaction between two surfaces involves the electrostatic double layer force, the Van der Waals force and the oscillatory nature of solvation pressure. The Van der Waals and the solvation forces dominate when the separation between the surfaces is small. On the other hand, the electrostatic double layer repulsion is strong at larger separations.

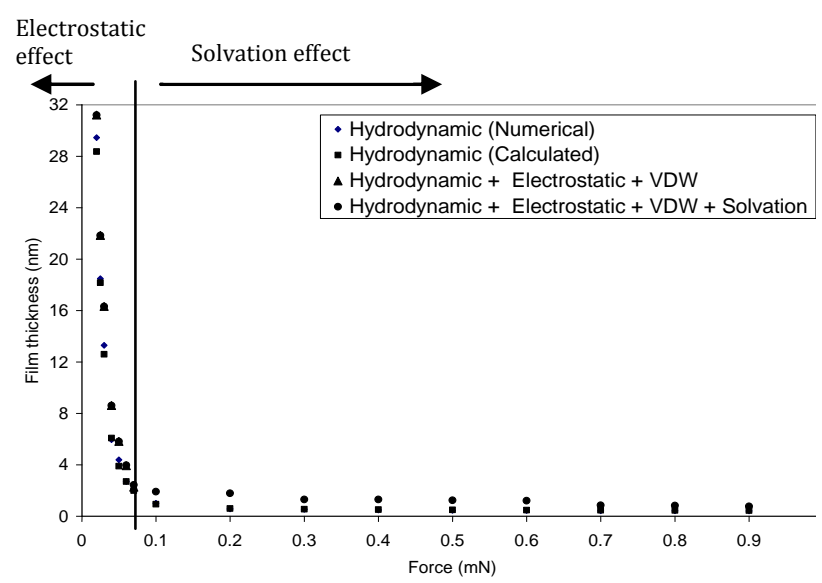

a) Variation of film thickness with load

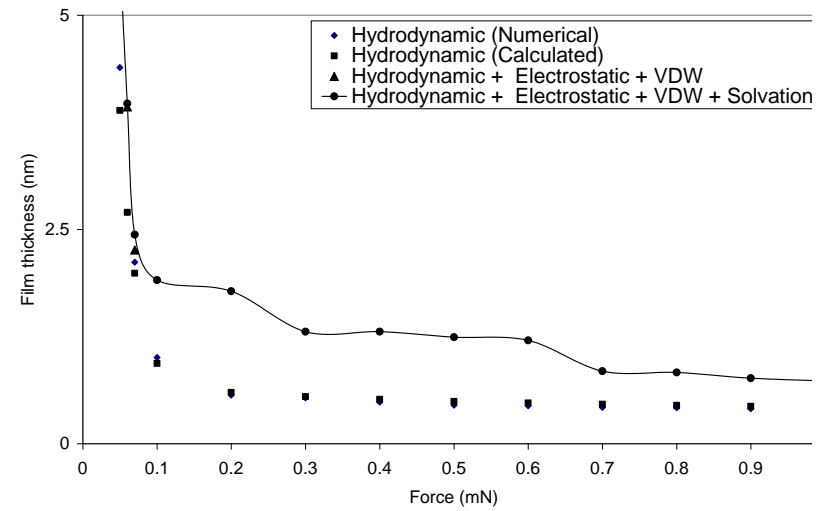

b) Enlarged of figure (a) for film thickness up to $5 \mathrm{~nm}$

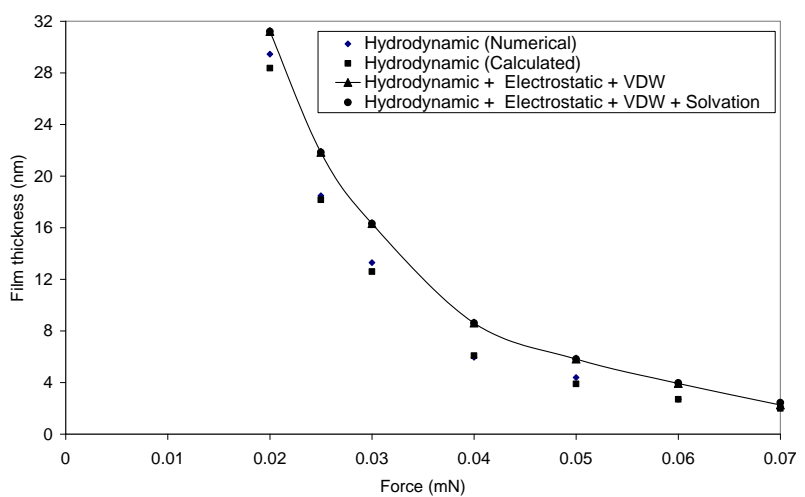

c) Enlarged of figure (a) for load up to of $0.07 \mathrm{mN}$

Fig. 7. Variation of film thickness with load for $U^{*}=$ $7.775 \times 10^{-16}$.

A clearer picture emerges, if for a given value of entraining speed, the applied load is changed to obtain the demarcation boundary between the region dominated by the surface force action of solvation, and that dominated by the electrostatic force in the formation of lubricant film thickness. Figure 7 (a) shows such a plot of film thickness against applied load for the value of entraining motion of $100 \mu \mathrm{m} / \mathrm{s}$ (i.e. $\mathrm{U}^{*}=7.775 \times 10^{-16}$ ). It should be noted that the layering effect (i.e. discretisation of the lubricant film) takes place by increasing the applied load from $0.07 \mathrm{mN}$ (Fig. 7 (b)) (i.e. $\mathrm{W}^{*}=1.944 \mathrm{X} 10^{-11}$ ). The interval of the discretized film thickness is about $0.5 \mathrm{~nm}$, which corresponds roughly to the molecular diameter of propylenecarbonate. This discretization is due to the solvation force which is observed when liquid molecules intervene in a narrow space between solid surfaces. The electrostatic effect gains in ascendancy by decreasing the applied load below about $0.07 \mathrm{mN}$ (Fig. 7 (c)). Generally, the film thickness obtained in both cases is larger than that the numerically predicted values or that calculated using either Brewe el al [27] or Hamrock and Dowson's [28] extrapolated oil film thickness equations, both for point contact geometries under iso-viscous elastic or isoviscous rigid regime of lubrication respectively.

Unfortunately, there exists no experimental or previous numerical work in the area of formation of ultra-thin film lubrication due to the effect of electrostatic double layer force to compare with the second set of results in this paper. This is an area requiring more research effort, and it is hoped that this initial investigation will induce further work.

\section{CONCLUSION}

In conclusion, it has been shown that the behavior of polar lubricants in concentrated contacts in ultra-thin conjunctions. The effect of hydrodynamic viscous force is relatively small as the intervening gap becomes one of several orders of magnitude of the molecular diameter of the intervening fluid and the effects of intermolecular forces of Van der Waals and solvation and electrostatic double layer force become significant. The results showed that at separation beyond about five molecular diameters of the intervening liquid, the net interaction is well described by continuum theories for the attractive Van der Waals force and the Poisson-Boltzmann equation for the 
repulsive double layer electrostatic force. The Van der Waals forces always dominate at sufficiently small separations (below about five molecular diameters of the intervening liquid) giving a force at molecular contact that is attractive (i.e. adhesion). But actually this is not the case, because under these conditions an additional solvation force is present that generally oscillate with distance, varying between attraction and repulsion, with a periodicity equal to the mean diameter of the liquid molecules. These results conform to the conclusions of previous works of Israelachvili [7,8], Israelachvili and Mcguiggan [9] and Christensen and Horn [10].

\section{Nomenclature}

a : Lubricant molecular diameter

A : Hamaker constant

$b \quad$ : Radius of Hertzian contact region

C : Constant defined in equation (11)

$D \quad$ : Deformation influence coefficient matrix

$E_{A, B} \quad$ :Young's modulus of elasticity

$E^{\prime} \quad$ : Reduced modulus of elasticity

$G^{*} \quad$ : Materials' parameter, $\mathrm{G}^{*}=\mathrm{E}^{\prime} \alpha$

$h \quad$ : Lubricant film thickness

$H \quad$ : Dimensionless film thickness, $H=h R / b^{2}$

$H_{0} \quad$ : Dimensionless central oil film thickness

$l \quad$ : Dimensionless side leakage boundary distance

$m$ : Dimensionless inlet distance

$n_{x}, n_{y}:$ Number of computational grid nodes

$P \quad$ : Total contact pressure

$p_{h} \quad$ : Hydrodynamic pressure

$p_{s} \quad$ : Solvation pressure due to surfaces' interaction force

$p_{v d w}$ : Pressure due to molecular Van der Waals' force

$P \quad$ : Dimensionless total contact pressure, $P=p / P_{\text {Her }}$

$P_{h} \quad$ : Dimensionless hydrodynamic pressure, $P_{h}=p_{h} / P_{\text {Her }}$

$P_{H e r}$ : Maximum Hertzian contact pressure

$P_{s} \quad$ : Dimensionless solvation pressure, $P_{s}=p_{s} / P_{H e r}$

$P_{V W} \quad$ : Dimensionless Van der Waals' pressure,

$P_{v d w} \quad P_{v d w}=p_{v d w} / P_{H e r}$

$N \quad$ : Total number of mesh points

$\mathrm{K} \quad$ : Elliptical ratio

$R \quad$ : Reduced radius of counterformal contact

$w \quad$ : Normal applied contact load

$W^{*} \quad$ : Load parameter, $W^{*}=w / E^{\prime} R^{2}$
$X, Y \quad$ : Dimensionless co-ordinates, $X=x / b, Y=y / b$

$U^{*} \quad$ : Speed (or Rolling Viscosity) parameter, $U^{*}=u \eta_{o} / E^{\prime} R^{2}$

$u \quad$ :Speed of entraining motion, $u=\left(u_{A}+u_{B}\right) / 2$

$Z \quad$ : Viscosity-pressure index

$\alpha \quad$ : Pressure of viscosity coefficient

$\delta \quad$ : Total elastic deformation

$\varepsilon, \xi \quad$ : Constants used in equation (4)

$\eta \quad$ : Lubricant dynamic viscosity

$\eta_{0} \quad$ : Atmospheric lubricant dynamic viscosity

$v \quad$ : Poisson's ratio

$\Omega \quad$ : Under-relaxation factor

$\rho \quad$ : Lubricant density

$\rho_{\mathrm{o}} \quad$ : Atmospheric lubricant density

$\bar{\rho} \quad$ : Dimensionless lubricant density, $\bar{\rho}=\rho / \rho_{0}$

$\bar{\eta} \quad$ : Dimensionless lubricant viscosity, $\bar{\eta}=\eta / \eta_{0}$ Superscripts:

$i, j \quad$ : Contravariant influence coefficient indices

$n \quad$ : Iteration index

Subscripts:

$A, B$ : Denote the contiguous bodies in contact

$k, l \quad$ : Covariant influence coefficient indices

\section{REFERENCES}

[1] C.H. Zhang, 'Research on Thin Film Lubrication: State of the Art', Tribology International, vol. 38, no. 4, pp. 443-448, 2005.

[2] J.B. Luo, S.Z. Wen and P. Huang, 'Thin Film Lubrication. part I: Study on the Transition Between EHL and Thin Film Lubrication Using Relative Optical Interference Intensity Technique', Wear, vol. 94, no. 1-2, pp. 107-115, 1996.

[3] J.B. Luo, P. Huang, S.Z. Wen and K.Y. Li, 'Characteristics of Fluid Lubricant Films at the Nano-Scale', Transaction ASME, Journal of Tribology, vol. 121, no. 4, pp. 872-878, 1999.

[4] J. Manojlović, 'Dynamics of SAMs in Boundary Lubrication', Tribology in Industry, vol. 35, no. 3, pp. 200-207, 2013.

[5] D. Tabor, 'Surface forces and surface interactions', Journal of Colloid and Interface Science, vol. 58, no. 1, pp. 2-13, 1977.

[6] J.N. Israelachvili and P.M. Mcguiggan, 'Adhesion and short range forces between surfaces. Part I: Part I: New Apparatus for Surface Force Measurement', Journal of Material Research, vol. 5, no. 10 , pp. $2223-2231,1990$. 
[7] J.N. Israelachvili, P.M. Mcguiggan and A.M. Homola 'Dynamic properties of molecularly thin liquid films', Science, vol. 240, no. 4849, pp. 189191, 1988.

[8] J.N. Israelachvili, 'Solvation forces and liquid structure, as probed by direct force measurements', Accounting Chemical Research, vol. 20, no. 11, pp. 415-421, 1987.

[9] J.N. Israelachvili, Intermolecular and surface forces. New York, $2^{\text {nd }}$ edition, Acdemaic Press, 1991.

[10] J.N. Israelachvili and P.M. Mcguiggan, 'Forces between surfaces in liquids', Science, vol. 241, no. 4867, pp. 795-800, 1988.

[11] H.K. Christensen and R.G. Horn, 'Direct measurement of the force between solid surfaces in a polar liquid', Journal of Chemical physical letters, vol. 98, no. 1, pp. 45-48, 1983.

[12] D.Y.C. Chan and R.G. Horn, 'The drainage of thin liquid films between solid Surface', Journal of Chemical Physics, vol. 83, no. 24, pp. 5311-5324, 1984.

[13] H. Matsuoka and T. Kato, 'An ultrathin liquid film lubrication theory-Calculation method of solvation pressure and its application to the EHL problem', Transaction ASME, Journal of Tribology, vol. 119, no. 1, 217-226, 1997.

[14] M.F. Al-samieh and H. Rahnejat, 'Nano-lubricant film formation due to combined elastohydrodynamic and surface force action under isothermal conditions', Proceedings Institution of Mechanical Engineering, Part C, vol. 215, no. 9, pp. 1019-1029, 2001.

[15] H. Matsuoka and T. Kato, 'Discrete nature of ulrathin lubrication film between mica surfaces', Transaction ASME, Journal of Tribology, vol. 118, no. 8, pp. 832-838, 1996.

[16] H. Matsuoka and T. Kato, 'Experimental study of ulrathin lubrication film thickness at the molecular scale', Proceedings of the Institution of Mechanical Engineering, Part J, vol. 211, no. 2, pp. 139-150, 1997.

[17] T. Kato and H. Matsuoka, 'Molecular layering in thin-film elastohydrodynamics', Proceedings of the Institution of Mechanical Engineering, Part J, vol. 213, no. 5, pp. 363-370, 1999.

[18] D. Dowson and G.R. Higginson, 'A numerical solution to the elastohydrodynamic problem',
Journal of Mechanical Engineering Science, vol. 1, no. 1, pp. 6-15, 1959.

[19] C.J.A. Roelands, 'Correlation aspects of viscositytemperature-pressure relationship of lubricating oils', PhD thesis, Delft University of Technology, The Netherlands, 1966.

[20] B.J. Hamrock and D. Dowson, 'Isothermal elastohydrodynamic lubrication of point contact. Part I- theoretical formulation', Transaction ASME, Journal of Tribology, vol. 98, no. 2, pp. 223-229, 1976.

[21] G. Horn and J.N. Israelachvili, 'Direct measurement of structural forces between two surfaces in a nonpolar liquid', Journal of Chemical Physics, vol. 75, no. 3, pp. 1400-1411, 1981.

[22] W. Van Megen and I. Snook, 'Solvent structure and solvation forces between solid bodies', Journal of Chemical Socity, Faraday Transaction II, vol. 75, pp. 1095-1102, 1979.

[23] A.M. Homola, J.N. Israelachvili, M.L. Gee and P.M. Mcguiggan, 'Measurements of and relation between the adhesion and friction of two surfaces separated by molecularly thin liquid films', Transaction ASME, Journal of Tribology, vol. 111, no. 3, pp. 675-682, 1989.

[24] E.M. Lifshitz, 'The theory of molecular attractive forces between solids', Soviet Physics, JETP, vol. 2, no. 1, pp. 73-83, 1956.

[25] E.J. W. Vewey and J. Overbeek, Theory of the stability of lyophobic colloids. Amsterdam, Elsevier, 1948.

[26] J.A. Greenwood, 'Presentation of elastohydrodynamic film thickness results', Journal of Mechanical Science, vol. 11, no. 2, pp. 128-132, 1969.

[27] D.E. Brewe, B.J. Hamrock and C.M. Taylor, 'Effect of geometry on hydrodynamic film thickness', Transaction ASME, Journal of Tribology, vol. 101, no. 2, pp. 231-239, 1979.

[28] B.J. Hamrock and D. Dowson, 'Elastohydrodynamic lubrication of elliptical contacts for materials of low elastic modulus, Part I- Fully flooded conjunction', Transaction ASME, Journal of Tribology, vol. 100, no. 2, pp. 236-245, 1978. 